\title{
FACTORES ASOCIADOS CON EL USO DE LOS PROTECTORES BUCALES EN DEPORTISTAS QUE PRACTICAN DEPORTES DE CONTACTO
}

\author{
${ }^{1}$ Mauricio Beleño Payares, ${ }^{1}$ Juan Bautista Merchán, ${ }^{1}$ Indira Mora Piedra, ${ }^{2}$ Guillermo Gómez M. \\ ${ }^{1}$ Estudiante de X semestre, F. de Odontología, U. Santo Tomás. \\ ${ }^{2}$ Medico Cirujano U. Industrial de Santander, Magister en Epidemiología Clínica U. Javeriana, \\ Docente U. Santo Tomás
}

Autor responsable de correspondencia: Guillermo Gómez $M$.

Dirección de correo electrónico: gomgui27@hotmail.com

\section{RESUMEN}

Objetivo: Identificar los factores asociados con el uso de los protectores bucales en practicantes de deportes de contacto.

Materiales y métodos: Se realizó un estudio experimental tipo ensayo clínico no controlado con 50 deportistas que practicaban deportes de contacto. En 25 de éstos se realizó un protector bucal. Para el análisis univariado se calcularon medidas de tendencia central. Para las variables se calcularon promedios y desviaciones estándar y para las cualitativas proporciones. En el análisis bivariado se empleo test de Chi2, test exacto de Fisher, t de student o test de rangos de Wilcoxon según la distribución de las variables y se consideró como variable de salida, la colocación o no del protector bucal.

Resultados: El 74\% (37) eran hombres, el promedio de edad fue entre $20.5 \pm 2.0$ años. El 54\% (27) pertenecían al equipo de fútbol y el $46 \%$ al de taekwondo. De los factores asociados con el uso del protector bucal en el grupo intervenido, se observó asociación estadísticamente significativa $(\mathrm{p}<0.014)$ con relación a la variable dificultad para comunicarse, hablar, gesticular, vociferar. Cabe resaltar que la dificultad para respirar con el protector bucal fué ligeramente superior para el grupo intervenido que utilizaban el protector bucal $(3.0 \pm 2.5)$.

Conclusiones: Ochenta por ciento de los deportistas de 18 a 25 años de edad, conocían o alguna vez les hablaron de los protectores bucales pero no los habían usado. [Beleño M, Merchan JB, Mora I, Gómez G. Factores asociados con el uso de protectores bucales en deportistas que practican deportes de contacto. Ustasalud Odontología 2008: 7: 21 - 27 ]

Palabras clave: Trauma dental, Deportes de contacto, Protector bucal.

\section{FACTORS ASSOCIATED WITH THE USE OF MOUTHGUARDS IN SPORTS PEOPLE WHO PRACTICE CONTACT SPORTS}

\begin{abstract}
Objective: To identify the factors associated with the use of the mouthguards in sports people who practice contact sports in the Santo Tomas University.

Materials and methods: An experimental controlled clinical study was made in parallel with 50 sportsmen and women who practiced contact sports. In 25 of these a mouthguard was used. For the univaried analysis measures of central tendency were calculated. The quantitative variables and the qualitative ones were analyzed with proportions. In the analysis bivaried were used Chi2, exact test of Fisher, $t$ of student or test of ranks of Wilcoxon according to the distribution of the variables.

Results: Thirty seven (74\%) were men; the average of age was between $20.5 \pm 2.0$ years. Twenty seven (54\%) belonged to the football team and $46 \%$ to the taekwondo. Of the factors associated with the use of the mouthguards, statistically significant association was observed $(\mathrm{p}<0.014)$ related to the variable difficulty to communicate, to speak, to gesture, to vociferate. It is possible to stand out that the difficulty to breathe with the slightly superior mouthguard was taken in the experimental group (3.0 \pm 2.5$)$.

Conclusions: Eighty percent of the sports people of 18 to 25 years of age knew or sometimes heard about the mouthguards but they had not used them.
\end{abstract}

Key words: Dental trauma, Contact sports, Mouthguards.

Recibido para publicación: 15 de febrero de 2008. Aceptado para publicación: 8 de marzo de 2008 . 


\section{INTRODUCCIÓN}

Las lesiones traumáticas en odontología generan mucho estrés tanto en el paciente como en las personas que lo acompañan, por esto, el odontólogo debe controlar la situación y calmar tanto al paciente como a sus padres u acompañantes; de igual forma, debe tomarse el tiempo necesario para realizar una evaluación minuciosa de las lesiones, de lo contrario, es probable que se pasen por alto lesiones significativas.

Uno de los factores de riesgo que se encuentran muy a menudo es el de las prácticas deportivas. Un importante sector de la población desarrolla actividades físicas individuales o colectivas en forma metódica desde la infancia y durante la adolescencia, etapas en las que se acentúa el crecimiento maxilofacial del individuo. ${ }^{2}$

La acción de los golpes suele causar la rotura, pérdida y hasta el desplazamiento de los dientes superiores e inferiores, situación que compromete además de la estética, la fonación y la masticación. El uso de los protectores bucales evita lesiones que afecten la lengua, encías, carrillo y labios, también expuestos a los impactos. Hoy en día, se pueden realizar estos protectores con materiales muy flexibles, consistentes e higiénicos. Se logra una adherencia perfecta a la anatomía bucal y se evitan las dificultades respiratorias. $^{3}$

Las personas tienen poca información sobre los beneficios que aportan los protectores bucales. Es importante promocionar y generar su uso en escolares $\mathrm{y}$ en adolescentes que realizan deportes de contacto para prevenir y/o disminuir las secuelas de lesiones traumáticas.

\section{El trauma dentoalveolar:}

El trauma dental ha sido y es un factor agravante en las alteraciones de la dentición en niños, adolescentes y adultos. Éstos se ven afectados, no solamente durante el trauma, sino también después de éste. Entre las complicaciones más frecuentes están: reabsorción radicular, pérdida de espacio, pigmentación dental, necrosis pulpar, pérdida prematura de dientes, laceraciones de tejidos blandos, problemas funcionales, estéticos y psicológicos entre muchos otros. En algunos casos causan daños irreparables no solamente en el momento del accidente, también durante $\mathrm{y} / 0$ postratamiento. ${ }^{4}$

En Colombia, el $17.6 \%$ de los pacientes que acuden a los servicios de urgencias odontológicas reportan fracturas dentales. Las lesiones de dientes se presentan con mayor frecuencia, en el grupo de 7 a 15 años de edad. ${ }^{5}$
A nivel regional se realizó un estudio de prevalencia de trauma dentoalveolar en la Clínica Carlos Ardila Lulle, en el período entre 1998 y 2002. De 1235 historias clínicas evaluadas, 116 casos correspondían a casos de trauma dentoalveolar con un promedio de edad de 18 años. ${ }^{6}$

En un estudio realizado en la Universidad de Yeditepe (Turquía) se observó que los hombres son más propensos a los traumas dentales que las mujeres. Las caídas eran el tipo de trauma más frecuente con un riesgo de complicación alto. ${ }^{7}$ Según Rabat Lamis, la principal causa de las lesiones dentales son por caídas $49.9 \%$, seguida por golpes con objetos inanimados $7.30 \%$, deportes $8.7 \%$ y violencia $7.7 \%{ }^{2}$

Sin embargo, otro estudio donde se evaluó el perfil de los pacientes con trauma dentoalveolar que asistieron a la sala de emergencia del Hospital Universitario de Sao Pablo, se encontró que de los 1650 pacientes que se presentaron, el 78 (4.7\%) presentaron trauma dental. Entre los factores etiológicos, las causas más frecuente fueron las caídas (40.8\%), seguido por accidentes automovilísticos (37.4\%); los accidentes deportivos correspondieron a un $3.4 \%{ }^{8}$

En relación con los deportes de alto riesgo, se ha observado que las lesiones ocurren principalmente durante la práctica de futbol, baloncesto, jockey, boxeo, artes marciales y taekwondo. Se encontró también que de los participantes de cualquier actividad deportiva, el $27 \%$ relató lesiones dentales $\mathrm{u}$ orofaciales; baloncesto y fútbol fueron los deportes con los más altos porcentajes de participantes lesionados. ${ }^{9}$

\section{Protector bucal como factor preventivo del trauma dental}

Los protectores bucales son necesarios por diferentes razones:

- Las lesiones dentales son las más frecuentes dentro de las lesiones orofaciales durante la práctica de deportes.

- La mayoría de estas lesiones se pueden prevenir.

- Más de 5 millones de dientes se pierden cada año, siendo la causa más frecuente (13\%-39\%) las producidas por traumatismos deportivos.

- Un atleta es 60 veces más susceptible de sufrir una lesión dentofacial cuando no lleva un protector bucal.

- Deportes considerados de riesgo medio, son los que presentan un número mayor de lesiones 
dentofaciales, mientras que otros deportes de mayor riesgo (hockey, boxeo, baloncesto, fútbol) presentan un número menor. Esto se debe a que el uso del protector bucal es obligatorio en el segundo grupo y no en el primero.

- El costo de un diente fracturado suele ser mayor que la confección de un protector bucal, y no sólo en el aspecto económico sino también en el aspecto psicosocial. ${ }^{2}$

- El protector bucal tiene un papel importante adicional cuando hay lesiones de cuello y conmoción debido a que amortigua los golpes en el mentón, en la zona anterior y en la región temporal del cráneo. Champán sugirió que si se usa el protector bucal, la posición condilar se sitúa $2 \mathrm{~mm}$ más adelante o alejada de lo normal de la base del cráneo cuando la boca está cerrada, se reduce la fuerza generada por un golpe ya que el protector la absorbe y la dispersa; de esta manera, se reduce el riesgo y la severidad daño a las estructuras condilares y a los tejidos de la base del cráneo. . $^{1,3,40}$

Se recomienda usar un protector bucal en los deportes en que existe el riesgo del contacto con los otros participantes o con superficies duras, tales como: baloncesto, softball, lucha grecorromana, fútbol, rugby, patinaje y artes marciales. ${ }^{11}$

El objetivo de este estudio fue Identificar los factores asociados con el uso de los protectores bucales en practicantes de deportes de contacto.

\section{MATERIALES Y MÉTODOS}

Se realizó un estudio experimental tipo ensayo clínico no controlado, el universo lo conformaron 130 deportistas que practicaban deportes de contacto como fútbol y taekwondo. Las personas que cumplieron con los criterios de inclusión establecidos en el estudio fueron 34 practicantes de taekwondo y 66 jugadores de fútbol para un total de 100 deportistas. La muestra la integraron 50 jugadores de ambos géneros que fueron seleccionados por un muestreo no probabilístico por conveniencia.

\section{Criterios de inclusión}

- Individuos que practican deportes de contacto como fútbol y taekwondo de la Universidad Santo Tomás, sede Floridablanca.

- Individuos que no utilizan protectores bucales en las prácticas deportivas.

- Individuos entre los 18 a 25 años de edad.

- Individuos que realicen sus prácticas deportivas en el primer semestre académico del año 2007.

\section{Criterios de exclusión}

- Individuos que tengan enfermedades respiratorias como asma o individuos que sean respiradores orales.

- Individuos que tengan alteraciones en la articulación temporomandibular (ATM).

- Individuos que padezcan enfermedades del sistema nervioso central como convulsiones.

- Individuos que utilicen aparatologia ortodóntica.

- Individuos que tengan problemas de oclusión severa.

\section{Las variables estudiadas fueron:}

Variables sociodemográficas: se registró el género, edad, tipo de deporte practicado.

Variables de conocimiento: se analizó si conocían el significado del protector bucal y si habían tenido posibilidad de usarlo

Variables relacionadas con el uso del protector bucal: se analizaron los factores asociados al uso del protector bucal durante las prácticas deportivas (dificultad para respirar, dificultad para hablar, nauseas, entre otros y que fueron recolectadas mediante escala análoga visual).

Variable intervención: la colocación o no del protector bucal

Se realizó un examen clínico individual para evaluar y determinar la población del estudio. Se descartaron individuos de acuerdo a los criterios de exclusión; se seleccionaron los 50 individuos de ambos géneros que participaron en el estudio.

A la totalidad de la muestra, 50 jugadores, se les pidió que diligenciaran el consentimiento informado y se les aplicó una encuesta de conocimiento general acerca del protector bucal. Posteriormente, se dividió la muestra en dos grupos conformados cada uno por veinticinco deportistas, 12 practicantes de Taekwondo y 13 jugadores de fútbol que se seleccionaron por conveniencia para cada grupo. Grupo 1 (25 jugadores), llamado grupo de estudio o intervenido y el grupo 2 o grupo control (25 jugadores).

Se procedió a ubicar a los participantes en los lugares de entrenamiento, luego se habló con cada instructor para que se dispusieran 15 minutos antes de empezar la práctica y exponer el proyecto. También se dieron charlas de prevención del trauma dental por medio de los protectores bucales durante las prácticas deportivas y de sus beneficios.

Se tomaron impresiones del maxilar superior al grupo 1 (intervenido). Los vaciados se realizaron 
en yeso tipo III (Whip Mix) para obtener modelos de trabajo de la arcada dental superior de cada atleta. Los protectores bucales se realizaron con una lámina de silicona de $4 \mathrm{~mm}$ de grosor aproximadamente, se probó la adaptabilidad del protector en boca. Luego se entregaron los 25 protectores a cada deportista para usarlo durante las prácticas deportivas por un periodo de tres semanas.

Se les dieron instrucciones a los jugadores sobre el mantenimiento y limpieza de los protectores bucales para que conservaran el protector en buen estado finalizada cada práctica deportiva

El proceso de evaluación del protector intraoral se realizó por medio de encuestas, al finalizar las tres semanas de la prueba, una encuesta para el grupo intervenido y otra para el grupo control, para determinar algunas de las variables como son: resequedad oral durante la práctica deportiva y facilidad para respirar.

Para saber si los deportistas estaban utilizando el protector bucal y asegurar el uso del mismo, se le indicó al instructor de cada deporte que el dispositivo fuera usado estrictamente como requisito durante las prácticas deportivas.

Para el análisis univariado las variables se describieron según medidas de resumen: para variables cualitativas se calcularon frecuencias y proporciones; para variables cuantitativas se calcularon medidas de tendencia central como la media y la mediana, desviación estándar.

Para el análisis bivariado se aplicaron pruebas estadísticas según la naturaleza de las variables para establecer asociaciones entre el grupo experimental y el control, con las variables cualitativas se aplicó la prueba estadística $\mathrm{Chi}^{2}$ o Exacto de Fisher; con las variables cuantitativas se aplicó prueba de t de Student o Test de Rangos de Wilcoxon dependiendo de la distribución de datos. Se consideró el valor de $\mathrm{p}=0.05$.

De acuerdo con la resolución número 008430 del 4 de octubre de 1993 del Ministerio de Salud, este trabajo se clasificó como una investigación de riesgo mínimo por prevalecer los criterios de respeto a la dignidad y los derechos de los pacientes sujeto de estudio.

\section{RESULTADOS}

Se evaluaron 50 deportistas que practican deportes de contacto en la Universidad Santo Tomas, sede Floridablanca y en el 50\% (25) de ellos se le realizó el protector bucal. El 74\% (37) eran hombres, el promedio de edad fue $20.5 \pm 2.0$ años. El 54\% (27) pertenecían a la selección de fútbol y el $46 \%$ a la de taekwondo. Al analizar las variables sociodemográficas relacionadas no se observó ninguna asociación estadísticamente significativa con relación a la edad, género y al deporte que se practica; concluyéndose que para estas variables el grupo intervenido y el grupo no intervenido presentaban características similares (Tabla 1).

Se demostró que todos los deportistas saben la definición del concepto del protector bucal, sin embargo, sólo el 30\% recibieron alguna vez información detallada de la importancia de usar los protectores en la prevención del trauma dental. El 84\% reportó tener conocimiento de la función que éste cumple pero no lo utilizaban y el 14\% reportó haberlo usado alguna vez en su vida. No se evidenciaron asociaciones estadísticamente significativas entre las variables de conocimiento, sin embargo la variable de conocimiento sobre qué es un protector bucal fue ligeramente superior en el grupo intervenido (60\%) con respecto al grupo no intervenido que fue de $40 \%$ (Tabla 2).

No hubo diferencia estadísticamente significativa entre los grupos, en relación con el reporte de algún tipo de lesión, sólo el 18\% (9) de los 50 deportistas reportó tener algún tipo de lesión. El único factor que influyó en el uso del protector bucal fue el de comunicación (3.4 \pm 2.9 ). De los factores asociados con el uso del protector bucal en el grupo intervenido, se observó asociación estadísticamente significativa $(\mathrm{p}<0.014)$ con relación a la variable dificultad para comunicarse, hablar, gesticular, vociferar con el protector bucal (4.6 \pm 2.9$)$. Cabe resaltar que la dificultad para respirar con el protector bucal fué ligeramente superior para el grupo intervenido que utilizaban el protector bucal $3.0 \pm 2.5$ (Tabla 3).

\section{DISCUSIÓN}

Estudios de investigación anteriores que examinan el uso de los protectores bucales en varios deportes, comparten la creencia que cuando son llevados correctamente reducen significativamente la cantidad y la severidad de lesiones orofaciales durante las practicas y competencias deportivas. Aunque los resultados de estos estudios han demostrado que cumplen la función de proteger, la mayoría de los jugadores se los retira durante la práctica sin darle el uso frecuente que este amerita. ${ }^{12}$

En la mayoría de los estudios revisados, se observó que los integrantes de estas investigaciones utilizaban protectores prefabricados que tienen mayores desventajas debido a que no son adaptados a las estructuras anatómicas de los deportistas y algunos protectores presentan materiales muy rígidos. Los 
protectores realizados sobre modelos con materiales blandos y flexibles como las siliconas han sido los de mayor aceptabilidad, adaptabilidad y comodidad para los atletas; además, presentan mejores propiedades físicas y mecánicas. Por lo tanto, en este estudio se realizaron los protectores de este tipo. ${ }^{13}$

En este estudio se demostró que los jugadores tienen un conocimiento informal de qué es y la fun- ción que cumple el protector bucal, sólo han escuchado alguna vez hablar de él a familiares, amigos, medios de comunicación, y hasta a los entrenadores de forma superficial, siendo esto un problema de política pública a nivel institucional, departamental y nacional porque el trauma dental en deportistas es una de consulta frecuente que se puede prevenir si hay una mayor capacitación de los entrenadores y de los deportistas.

Tabla 1. Descripción de las variables sociodemográficas y tipo de deporte, en grupo intervenido y no intervenido.

\begin{tabular}{|c|c|c|c|c|}
\hline \multirow[b]{2}{*}{ Variables } & \multirow{2}{*}{$\begin{array}{c}\text { Global } \\
\text { Frecuencia (\%) }\end{array}$} & \multicolumn{2}{|c|}{ Intervenidos } & \multirow[b]{2}{*}{$\mathrm{P}^{*}$} \\
\hline & & $\begin{array}{c}\text { Si } \\
\text { Frec. }(\%)\end{array}$ & $\begin{array}{c}\text { No } \\
\text { Frec. (\%) }\end{array}$ & \\
\hline Global & $50(100)$ & $25(50)$ & $25(50)$ & \\
\hline \multicolumn{5}{|l|}{ Género } \\
\hline Hombre & $37(74)$ & 19 (51.3) & $18(48.6)$ & $0.747^{*}$ \\
\hline Mujer & $13(26)$ & $6(24)$ & 7 (28) & \\
\hline Edad (¥) & $20.5 \pm 2.0$ & $20.5 \pm 2.1$ & $20.4 \pm 2.0$ & $0.8347^{* *}$ \\
\hline \multicolumn{5}{|c|}{ Tipo de deporte } \\
\hline Fútbol & $27(54)$ & $13(48.1)$ & $14(51.8)$ & $0.777^{*}$ \\
\hline Taekwondo & $23(46)$ & $12(52.2)$ & $11(47.8)$ & \\
\hline
\end{tabular}

${ }^{*}$ Test de chi cuadrado ${ }^{* *}$ Prueba $t$ de Student $\mathrm{p}=0.05$,

(¥) Variable cuantitativa: presenta promedio y desviación estándar

Tabla 2. Descripción del conocimiento acerca de los protectores bucales según grupo intervenido y no intervenido.

\begin{tabular}{|c|c|c|c|c|}
\hline \multirow{2}{*}{ Variables } & \multirow{2}{*}{$\begin{array}{l}\text { Global } \\
\mathrm{n}(\%)\end{array}$} & \multicolumn{2}{|c|}{ Intervenidos } & \multirow[t]{2}{*}{$\mathrm{P}^{*}$} \\
\hline & & $\begin{array}{c}\mathrm{Si} \\
\mathrm{n}(\%)\end{array}$ & $\begin{array}{c}\text { No } \\
\mathrm{n}(\%)\end{array}$ & \\
\hline \multicolumn{5}{|l|}{ Concepto del protector bucal } \\
\hline $\mathrm{Si}$ & $50(100)$ & $25(50)$ & $25(50)$ & --- \\
\hline \multicolumn{5}{|c|}{ Conocimiento sobre que es un protector bucal } \\
\hline No & $10(20)$ & $6(60)$ & $4(40)$ & $0.480^{*}$ \\
\hline $\mathrm{Si}$ & $40(80)$ & $19(47.5)$ & $21(52.5)$ & \\
\hline \multicolumn{5}{|c|}{$\begin{array}{l}\text { Conocimiento sobre la función que cumple el } \\
\text { protector bucal }\end{array}$} \\
\hline No & $8(16)$ & $4(50)$ & $4(50)$ & $1.0^{*}$ \\
\hline $\mathrm{Si}$ & $42(84)$ & $21(50)$ & $21(50)$ & \\
\hline \multicolumn{5}{|c|}{ Información sobre que es un protector bucal: } \\
\hline No & $35(70)$ & $17(48.6)$ & $18(51.4)$ & $0.758^{* *}$ \\
\hline $\mathrm{Si}$ & $15(30)$ & $8(53.3)$ & $7(46.7)$ & \\
\hline \multicolumn{5}{|c|}{ Posibilidad del uso del protector bucal } \\
\hline No & $43(86)$ & $22(51.2)$ & $21(48.8)$ & $0.684^{*}$ \\
\hline $\mathrm{Si}$ & $7(14)$ & $3(42.9)$ & $4(57.1)$ & \\
\hline
\end{tabular}

* Test Exacto de Fisher, **Test de chi cuadrado, $\quad \mathrm{p}=0.05$ 
Tabla 3. Descripción de los factores asociados durante las prácticas deportivas, según grupo intervenido y no intervenido.

\begin{tabular}{|c|c|c|c|c|}
\hline \multirow[b]{2}{*}{ Variables } & \multirow{2}{*}{$\begin{array}{l}\text { Global } \\
\text { n (\%) }\end{array}$} & \multicolumn{2}{|c|}{ Intervenidos } & \multirow[t]{2}{*}{$\mathbf{P}$} \\
\hline & & $\begin{array}{c}\mathrm{Si} \\
\mathrm{n}(\%)\end{array}$ & $\begin{array}{c}\text { No } \\
\mathrm{n}(\%)\end{array}$ & \\
\hline \multicolumn{5}{|l|}{ Lesiones causadas } \\
\hline No & $41(82)$ & $19(46.3)$ & $22(53.7)$ & \multirow{2}{*}{$0.463^{*}$} \\
\hline $\mathrm{Si}$ & $9(18)$ & $6(66.7)$ & $3(33.3)$ & \\
\hline \multicolumn{5}{|l|}{ Comodidad del protector bucal } \\
\hline No & $23(92)$ & $23(92)$ & --- & \multirow[t]{2}{*}{---} \\
\hline $\mathrm{Si}$ & $2(8)$ & $2(8)$ & --- & \\
\hline Estabilidad del protector bucal (¥) & $2.3 \pm 2.5$ & $2.3 \pm 2.5$ & --- & --- \\
\hline $\begin{array}{l}\text { Dificultad para respirar con el protector } \\
\text { bucal (¥) }\end{array}$ & $2.5 \pm 2.4$ & $3.0 \pm 2.5$ & $1.96 \pm 2.3$ & $0.1361^{* *}$ \\
\hline $\begin{array}{l}\text { Dificultad para comunicarse, hablar, } \\
\text { gesticular, vociferar con el protector } \\
\text { bucal (¥) }\end{array}$ & $3.4 \pm 2.9$ & $4.6 \pm 2.9$ & $2.0 \pm 2.4$ & $0.0014^{* *}$ \\
\hline Náuseas ( $¥)$ & $1.6 \pm 2.0$ & $1.9 \pm 2.3$ & $1.3 \pm 1.6$ & $0.5575^{* * * *}$ \\
\hline $\begin{array}{l}\text { Resequedad oral durante la práctica } \\
\text { deportiva ( } ¥)\end{array}$ & $4.4 \pm 3.0$ & $4.3 \pm 3.0$ & $4.5 \pm 3.0$ & $0.7782^{* * * *}$ \\
\hline
\end{tabular}

${ }^{*}$ Test Exacto de Fisher, ${ }^{* *}$ Prueba T de student, ${ }^{* * *}$ Test de chi cuadrado, ${ }^{* * * *}$ Test de rangos de Wilcoxon $\quad \mathrm{p}=0.05$

(¥) Variable cuantitativa: presenta promedio y desviación estándar

Algunos resultados fueron inesperados, como la dificultad para respirar y la sensación de náuseas; los resultados con diferencia estadísticamente significativa, se dieron en la variable de la comunicación, hablar, gesticular, vociferar donde el grupo intervenido mostró un promedio de $4.6 \pm 2.9$ con referencia al grupo control $(p=0.0014)$. Los resultados indicaron que no hay una diferencia amplia entre usar y no usar el protector bucal, por lo tanto los factores asociados al uso (náuseas, dificultad para respirar, dificultad para hablar, entre otros) se pueden disminuir de forma significativa al generar un hábito de uso del protector desde que los deportistas sean niños y adolescentes, para que se adapten a éste.

Cabe resaltar que en la revisión de la literatura, la totalidad de los artículos encontrados son de países desarrollados europeos y norteamericanos, pues en nuestros países latinoamericanos hay muy poca información sobre los protectores bucales. Se requiere un programa de promoción y prevención dirigido a los entrenadores de las diferentes selecciones deportivas acerca de la frecuencia de trauma dental asociada a pacientes que practican deportes de contacto para resaltar la importancia del protector bucal y sus beneficios.

Sin embargo, es importante anotar que aunque no se han identificado programas orientados a prevenir el trauma dental dirigido a entrenadores deportivos; en la literatura revisada se logró demostrar la eficacia del uso de un material educativo dirigido a docentes de educa- ción básica que ilustra sobre el manejo de la avulsión dental y que demuestra que este tipo de estrategias podrían ser alternativas viables que favorezcan las acciones orientadas a promover la salud bucal y prevenir el trauma dental en las comunidades escolarizadas. ${ }^{14}$

Al considerar las implicaciones prácticas de este trabajo es importante anotar que de acuerdo a lo expuesto por Brito y Col, los estudios han demostrado que es importante incluir a los profesores de las asignaturas de educación física en los procesos orientados al manejo y prevención del trauma orobucofacial. En este trabajo y debido a la intervención realizada se logró un efecto que puede multiplicarse más allá de la población intervenida; pues la mayoría de estos jóvenes son personas vinculadas al programa de cultura fisica de la USTA aspecto que podría incidir en lograr que esta conducta de autocuidado se difunda mejor. ${ }^{14}$

En esta investigación se encontró que el $80 \%$ de los deportistas de 18 a 25 años de edad, conocían o alguna vez les hablaron de los protectores bucales, comparado con el $78 \%$ encontrado en otro estudio que evaluó las actitudes y el conocimiento de los jugadores que practican deportes de contacto al llevar el protector bucal. ${ }^{12}$

\section{BIBLIOGRAFÍA}

1. Crona-Larsson G, Norén JG. Luxation injuries to permanent teeth - a retrospective study of etiological factors. Dental Traumatol 1989; 5: $176-179$. 
2. Cornwell H, Messer LB, Speed H. Use of mouthguards by basketball players in Victory, Australia. Dental Traumatol 2003; 19: 193 - 203.

3. Levin L, Friedlender LD, Gerner SB. Dental and oral trauma and mouthguard use during sport activities in Israel. Dental Traumatol 2003; 19: 237- 242.

4. Rajab LD. Traumatic dental injuries in children presenting for treatment at the Department of Pediatric Dentistry, Faculty of Dentistry, University of Jordan 19972000. Dental Traumatol 2003; 19: 6 - 11.

5. República de Colombia. Ministerio de Salud. III Estudio Nacional de Morbilidad Oral (ENSAB III). p. 199.

6. León JC, Contreras E, Pineda L, Galeano CF. Prevalencia del trauma dentoalveolar en pacientes atendidos en el servicio de urgencias de la Clínica Carlos Ardila Lulle de Floridablanca - Colombia, entre 1998 y 2002. Ustasalud Odontología 2004; 3: 32 - 40.

7. Sandalli N, Cildir S, Guler N. Clinical investigation of traumatic injuries in Yeditepe University, Turkey during the last 3 years. Dent Traumatol 2005; 21: 188 - 194.

8. Rezende FM, Gaujac C, Rocha AC, Peres MPS. A prospective study of dentoalveolar trauma at the Hospital das Clínicas, São Paulo University Medical School. Clinics 2007; 62: 133 - 138.

9. Traebert J, Peres MA, Blank V, Böell Rda S, Pietruza JA. Prevalence of traumatic dental injury and associated factors among 12 year old school children in Florianopolis, Brazil. Dental Traumatol 2003; $19: 15-18$.

10. Chalmers DJ. Mouthguards protection for the mouth in rugby union. Sports Med 1998; 25: 339 - 349.

11. Newsone PR, Tran DC, Cooke MS. The role of the mouthguard in the prevention of sports - related dental injuries: a review. Int J Paediatr Dent 2001; 11: 396 - 404.

12. Miller M, Gariepy G, Tittler J, Berry D. Attitudes of High school ice hockey players toward mouthguard usage. The Internet Journal of Allied Health Sciences and Practice. October 2006. Volume 4. Number 4. [en línea] fecha de acceso: junio de 2007. URL disponible en: http://ijahsp. nova.edu/articles/vol4num4/miller.pdf

13. Holmes C. Mouth protection in Sport in Scotland - a review. Br Dent J 2000; 188: 473 - 474.

14. Brito CP, Garnica MJ, Rincón ML, Moreno JO. Implementación y validación de un material educativo orientado a promover el conocimiento y manejo de la avulsión dental de dientes permanentes en docentes de básica primaria y bachillerato de instituciones públicas y privadas de Bucaramanga, Santa Marta y Riohacha. Ustasalud Odontología 2006; 5: 107 $-114$
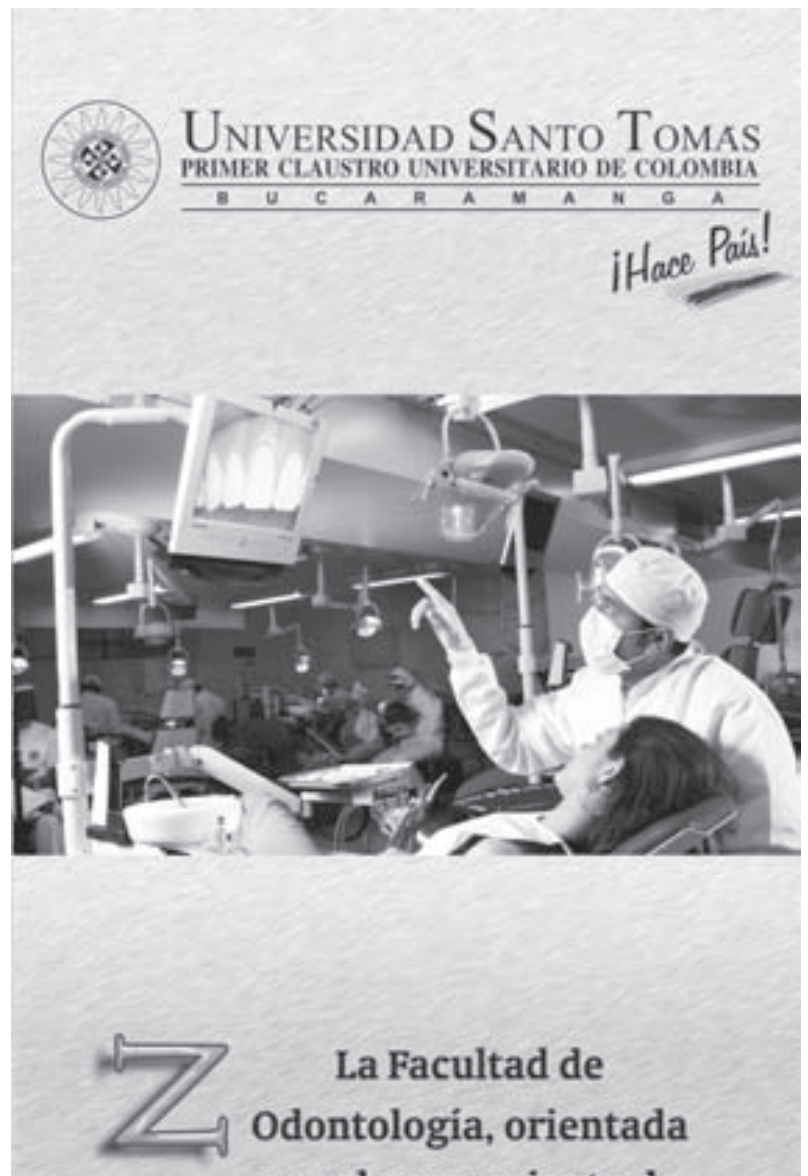

La Facultad de Odontologia, orientada por el pensamiento de Santo Tomás, pretende formar Odontólogos integrales y/o especialistas a través de parámetros

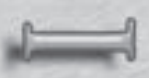

humanísticos, éticos, cientificos, biotecnológicos,

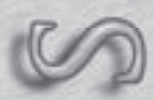
investigativos $y$ sociales, como recurso

humano capaz de

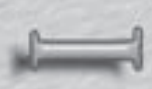
intervenir con éxito en el proceso dinámico de la salud y la enfermedad, en el individuo, la familia y la comunidad. 